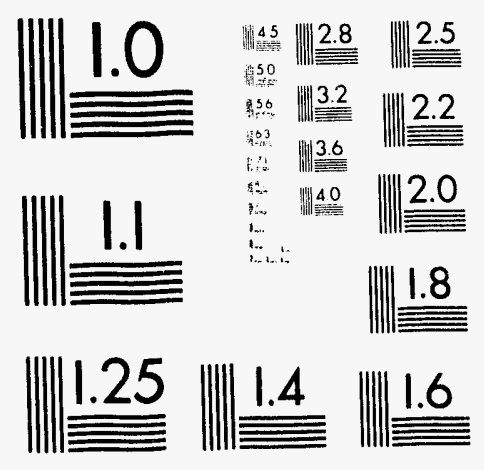



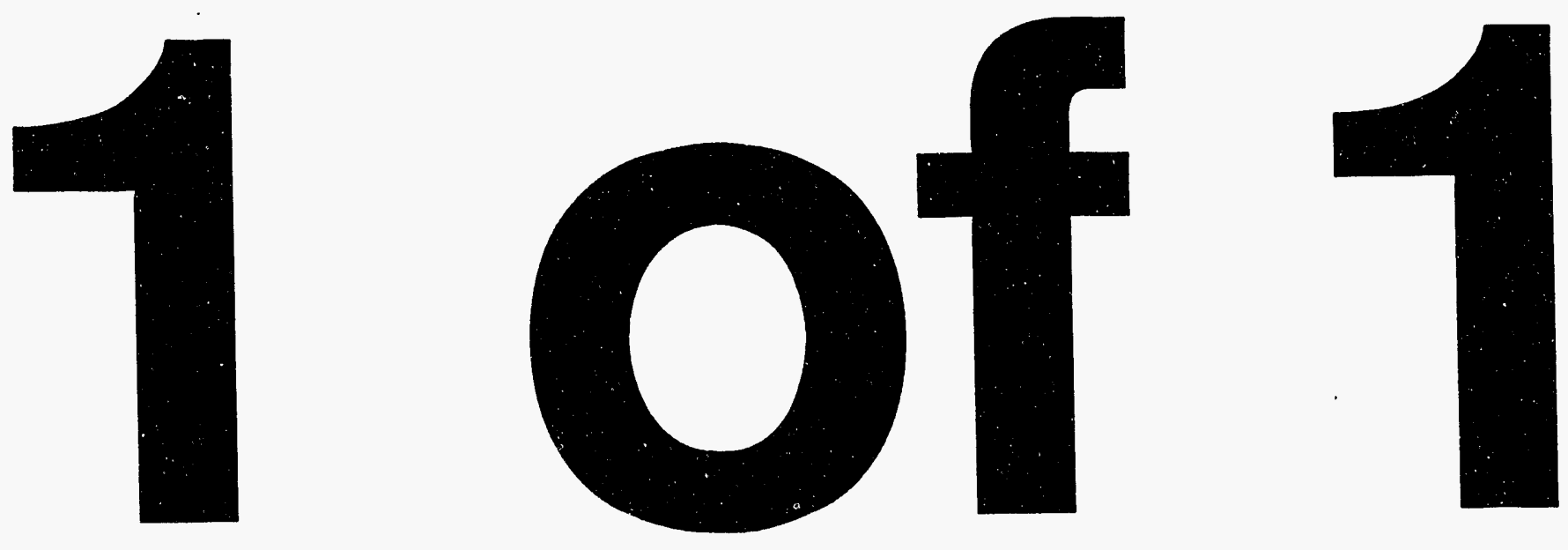


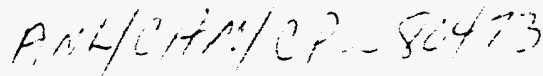 \\ Lit- $253 x^{2} i--2$}

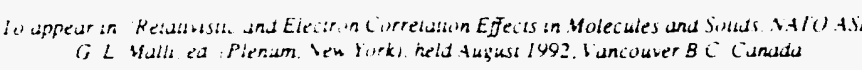

\section{A DISCUSSION OF SOME ASPECTS OF THE MCSCF METHOD}

\section{Ron Shepard}

Theoretical Chemistry C'roup

Chemistry Division

Argonne National Laboratiry

Argonne. Il 60439

\section{INTRODUCTION}

After a brief overview of the MCSCF (MultiConfiguration Self-Consistent-Field) method. a more detailed examination of the underlying model is undertaken. The problem of "multiple solutions" is examined with reference to the "symmetry-breaking" problem and with emphasis on single-state cases. It is argued that the most appropriate resolution of such problems is usually case-specific, and depends on the details of how the MCSCF such problems is usually case-specific, and depends on the details of how the MCSCF
wave function is being used to model the chemical system rather than on features wave function is being used to model the chemical system rather than on features
characteristic of the MCSCF wave funcrion itself. Finally, a recently introduced MCSCF wave function optimization method based on multidimensional trigonometric interpolation is briefly discussed.

\section{THE MCSCF METHOD}

There are several aspects of the MCSCF method that determine its usefulness, validity, and accuracy'. Firstly, the MCSCF wave function is based on the Born-Oppenheimer approximation. This approximation is the basis of the "potential energy surface (PES)" concept. Secondly, it makes use of finite orbital basis sets, although it would be possible to imagine an ideal MCSCF method involving a finite number of molecular orbitals that are imagine an ideal MCSCF method involving a finite number of molecular orbitals that are
arbitrarily flexible (i.e. as if they were constructed from an infinite, complete, orbital basis arbitrarily flexible (i.e. as if they were constructed from an infinite, complete, orbital basis
set). A conceptual and computational advantage of the former approach is that the MCSCF wave function may be compared to the full- $\mathrm{Cl}$ wave function in the same finite orbital basis, and in this sense it may be regarded as an approximation to this full- $\mathrm{Cl}$ wave function.

The MCSCF wave function is usually written as a linear superposition of configuration state functions (CSFs) $\left\{|\bar{n}\rangle, n=1 \quad v_{c s i}\right\}$

$$
\left.|m c\rangle=\sum_{n=1}^{\infty} c|j| \bar{n}\right\rangle
$$

although several nonlinear expansion forms have been employed also'. In a nonlinear expansion, the expansion coefficients $c_{n}$ depend in some (nonlinear) manner on a usually much smaller number of underlying variational parameters. This discussion will focus primarily on the linear expansion as given above. It is not uncommon for the MCSCF wave function to be expanded in terms of primitive Slater determinants. Such expansions are usually larger than the equivalent CSF expansion, due to the fact that the determinants span the space of several spin states, but it is sometimes argued that the simplicity of this approach compensates for this disadvantage. The MCSCF wave function approximates the approach compensates for this disadvantage. The MCSCF wave function approximates the Eq. (1). If all expansion terms were included, there would be no approximation, and in this sense the full-CI wave function is a special case of an MCSCF wave function. If the summation in Eq. (1) is limited to a single expansion term, this leads to the other limiting case of the MCSCF expansion, namely, the (single configuration) SCF wave function. The remaining flexibility of the MCSCF wave function arises from the variation of the shape of the orbials from which the expansion CSFs are constucted the MCSCF wave function atrempis 10 approximat attempts to approximate be regarded as the "exact" wave function, by the simultaneous variation
coefficients and of the orbitals from which the CSFs are constructed.

The MCSCF energy is generally taken to be the expectation value of the hamiltonian cperator

$$
E(m c)=\frac{\langle m c|H| m c\rangle}{\langle m c \mid m c\rangle}
$$

for a paricular choice of expansion coefficients and orbitals. This energy expression immediately introduces ambiguity in the definition of the wave function because the energy is independent of overali scaling of the CSF expansion coefficients in Eq. (I). This ambiguity is handied formally by imposing a normalization constraint on the expansion coefficients in the following way. A reference wave function, denoted as $|0\rangle$ and with expansion coefficients $\mathrm{c}^{0}$, is chosen which sausfies the nomalization $\langle 0 \mid 0\rangle=\left|c^{0}\right|^{2}=1$. For a given set of orbitals, variations of the trial wave function are then defined in terms of some arbitrary set of orthogonal expansion states $|n\rangle$ where $\langle n \mid 0\rangle=0$ and $\langle n \mid m\rangle=\delta_{\text {m. }}$ are satisfied. (Note that an expansion state $\mid n)$ as useci here is itself a linear expansion of CSFs $|\hat{n}\rangle$.) It is convenient to use the operator ${ }^{3,4}$

$$
P=\sum_{n=0} p_{n}(|n\rangle\langle 0|-| 0\rangle\langle n|)
$$

to define these variations. Note that $P|0\rangle$ is an arbitrary vector, with arbitrary normalization, within the orthogonal complement space, and that this vector is parameterized with $\left(N_{c s f}-1\right)$ parameters $p_{n}$. The trial wave function may then be written as

$$
|m c\rangle=\exp (P)|0\rangle
$$

where $\exp (P)$ is a unitary (or, for real transformations, orthogonal) operator. This parameterization results in the idenuty $\langle m c \mid m c\rangle=\langle 0| \exp (-P) \exp (P|| 0\rangle=\langle 0 \mid 0\rangle=1$, simplifying great!y the formal manipularions involving Eq. (2) by eliminating from further consideration the dependence of the denominator on the variational parameters.

It is possible to find also a similarly useful parameterization of the orbital vanations. For real orbital transformations. let

$$
\phi=\phi^{0} \mathrm{U}
$$

where the row vector $\phi^{0}$ is some orthonormal set of reference orbitals, and $\mathrm{U}$ is an orthogonal transformation matrix. The reference orbitals may be written in terms of a 
nonorthogonal atomic basis $x$ as $\bullet^{0}=x \mathrm{C}^{0}$, giving implicitly $\phi=x \mathrm{C}^{0} \mathrm{U}=\mathbf{x}$ where $\mathrm{C}=\mathrm{C}^{0} \mathrm{U}$. If the elements of the matrix $C$ are used directly to describe the orbital variations, then complications arise due to the nonorhogonal nature of the basis $\boldsymbol{X}$ and orbitals . One approach to address this issue is to introduce a large number. $\frac{1}{2} N_{\Delta \alpha}\left(N_{\Delta \alpha+e}+1\right)$. of Lagrange multipliers, and to replace Eq. (2) accordingly with a more general Lagrange functional in terms of these nonorthogonal orbitals. This approach is generally considered to be too restrictive for a general MCSCF procedure. Instead, it has been found quite practical to formulate the orbital variations using the following approach ${ }^{1-7}$

The matrix $U$ may be parameterized as $U=\exp (K)$ where the matrix $K$ is skewsymmetric ( $K^{\mathrm{T}}=-\mathbf{K}$, or in terms of matrix elements $K_{r s}=-K_{s r}$ ). An arbitrary subset of the $-N_{\alpha_{0}}\left(N_{y_{0}}-1\right)$ distinct matrix elements may be chosen to define $\mathbf{K}$. For convenience of notation, we hereafter assume that these are chosen as ihe strictly lower triangle matrix elements, $K_{r s}$ for $r>s$. For subsequent purposes, it will te convenient to define the vector $x$ to be the appropriate collection of these distinct parameters. This parameterization achieves a reduction in the number of energy-dependent orbital parameters, from

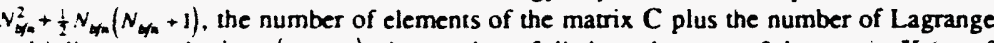
multipliers, to only $\frac{1}{2} N_{\text {wan }}\left(N_{-1}-1\right)$, the number of distinct elements of the matrix $K$ (or of the vector $x$ ). (For brevity, the additional reductions of the number of variational parameters for some wave function expansions due to redundant orbital rotations ${ }^{1}$ will be ignored in this discussion.) Since the elements of $\mathbf{K}$ are completely free to vary, and the simplified. In order to proceed further, the one-electron operator ${ }^{5} K$ is defined as

$$
K=\sum_{n=1} K_{n} E_{n}=\sum_{i=1} K_{n}\left(E_{n}-E_{m}\right)
$$

with $E_{\mathrm{z}}=a_{s}^{*} a_{s a}+a^{*} a^{0}$ being the spin-symmetry-adapted combination of products of spinorbital creation and annitilation operators. It is now relatively straightforward to show that an arbirary trial wave function in terms of the orbital basis is exactly equivalent to

$$
\mid m c, \phi)=\exp (K) \mid m c \cdot \phi \varphi
$$

It is imporant to emphasize that this expression is not an approximation, and that it is valid for arbitrary values of the parameters $K$. Using the $P$ operator parameterization for the MCSCF trial function finally gives

$$
\mid m c . \varphi)=\exp (K) \exp (P) \mid 0 ; \emptyset 9
$$

where the wave function $10 ; 0$ is the reference wave function consisting of the reference CSF coefficients $\mathrm{c}^{0}$ and of CSF expansion terms constructed from the reference orbital coefficients $\mathbf{C}^{0}$

With these conceprs in mind. we can now describe in more detail MCSCF wave functions and energy expressions. In terms of normalized CSF expansion coefficients, the tral energy may be written as

$$
\begin{aligned}
& E^{\text {inal }}=\mathbf{c}^{T} \mathbf{H}(\mathrm{K}) \mathbf{c} \\
& =\sum c_{-}\langle\dot{m}: 0|\mu| \tilde{n}: \oplus\rangle c_{0} \\
& \left.=\sum_{n=0} c_{-}\left(m: 0^{\circ} \mid \exp 1-K\right) H \exp K X \mid \bar{n}: 0^{0}\right\} c_{n}
\end{aligned}
$$

In $E_{4} .(10)$. the $K$ dependence is implicit in the hamiltonian matrix elements, whereas in Eq $(11)$ is is given explicitly in the orbital transtormation operator exp $(K)$. Agann, it should be stressed that Eqs 110$)$ and (11) are exactly equivalent; no approximations have yet been introduced. If the usual second quantized expression for the hamiltonian operator is used in these equations.

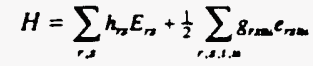

with $E_{0}=E_{n}-\delta E_{n}$ then these differences become more clear. Namely, all integrals, $h_{r s}$ and $g_{r s a s}$ are defined in Eq. (10) with respect to the trial orbital basis $\phi$, whereas they are defined in Eq. (11) with respect to the reference orbital basis $\varphi^{0}$. (The operators $E_{r s}$ and ersu are calied, respectively', the unitary group generators and normal order generator products.) Because this is the only essential difference between Eqs. (10) and (11), it is customary to associate this orbital dependence with the operator instead of with the CSF expansion basis. For example, the above energy expressions are writen

$$
\begin{aligned}
& E^{\text {tral }}=\sum_{\infty} c_{m}\langle\dot{m}|H| \bullet \mid \tilde{i}\rangle c_{.} \\
& =\sum_{-\infty} c_{-}\left(\bar{m}|\exp (-K) H|_{0}^{0}|\exp (K)| \bar{n}\right) c_{\infty}
\end{aligned}
$$

where the orbital dependence is denoted with square brackets. In this sense, the primitive expansion kets contain only the occupation and spin-coupling information. Eqs. (13) and (14) lead to the operator identiry

$$
H(\bullet)=\exp (-K) H\left(\bullet^{0} \exp (K)\right.
$$

which proves useful when ccasidering orbital variations. If this expression is expanded by orders in the matrix elements of $K$

$$
H[\bullet]=H^{0}+\left[H^{0}, K\right]+\frac{1}{2}\left[\left[H^{0}, K\right], K\right]+\ldots
$$

then a series of approximations may be developed for the $K$-dependent hamiltonian matrix elements of Eqs. $(10-14)$. Here, $H^{0}$ is used as a shorthand notation for $H\left[O^{2}\right]$. the hamiltonian operator in terms of the reference orbitals. For each of these approximations to $H[0]$, there is a corresponding approximation to the E$E^{\text {rrial }}$ expression of Eq. (13) that determines an approximate dependence of the trial energy on the orbital variations. These approximate hamiltonian operators may be written in the usual form of Eq. (12) where the effective integrals, which are the coefficients of the generators and generator products, may be obtained numerically using successive one-index transformations 1,8 .

When combined with the exp $(P)$ parameterization of the CSF coefficient changes, the trial energy may be writien as

$$
E^{\text {tral }}=\left\langle 0\left|\exp (-P) \exp (-K) H^{0} \exp (K) \exp (P)\right| 0\right\rangle
$$

When expanded in the elements of the matrix $K$ and vector $p$. Eq. (17) may be written as

$$
E^{\text {out }}=\left\langle 0\left|H^{0}+\left[H^{0} \cdot K\right]+\left[H^{0} \cdot P\right]+\left[\left[H^{0} \cdot K\right] \cdot P\right]+\frac{1}{2}\left[\left[H^{0}, K\right] \cdot K\right]+\frac{1}{2}\left[\left[H^{0} \cdot P\right] \cdot P\right]+\ldots\right| 0\right\rangle
$$

In practice, the infinite expansion of Eq. (18) is often truncated to include the second-order terms. and written in matrix notation as

$$
\left.E^{|2|} \mid \mathbf{x}, \mathbf{p}\right)=E(0)+\left(\begin{array}{ll}
\mathbf{x}^{T} & \mathbf{p}^{T}
\end{array}\right)\left(\begin{array}{l}
w \\
\mathbf{v}
\end{array}\right)+\frac{1}{2}\left(\begin{array}{ll}
\mathbf{x}^{T} & \mathbf{p}^{T}
\end{array}\right)\left(\begin{array}{cc}
\mathbf{B} & \mathbf{C} \\
\mathbf{C}^{T} & \mathbf{M}
\end{array}\right)\left(\begin{array}{l}
\mathbf{p} \\
\mathbf{p}
\end{array}\right)
$$

This expansion is useful for considering variations of both the orbitals and the expansion coefficients, but it is accurate only for small values of the individual parameters of the $x$ and $p$ vectors since it derives from a low-order truncation. In Eq. (19), $w$ and $v$ are the 

ortital and CSF gradient vectors respectuvely, and the matrices $\mathbf{B}$. C. and $\mathbf{M}$ are the matrix block $C$ and the ortital coefficient matn $x$ must be distinguished by context.:

If it is assumed that $0^{0}$ is an eigenvector of the matrix representation of $H^{0}$. then the CSF gradient vector $v$ vasishes from an expansion such as Eq. (19). This idea can be extended one step furher ${ }^{7}$, by imposing the condition on $p$ thas the energy maintain this minımization condition for arbitrary $\mathbf{K}$. Conceptually for a ground state wave function, this is equivalent to requiring that $\mathrm{c}$ of Eq. (13) is always the lowest eigenvector of the matrix representation of $H(\bullet)$. This trial energy may be denoted as

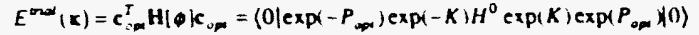

This condition establishes an implicit relationship of $\mathbf{p}$ on the matrix $\mathbf{K}$. denoted as $\mathbf{p}(\mathbf{K})$ is determined from the truncated Eq. (19), this relation is

$$
\mathbf{p}=-\mathbf{M} \cdot{ }^{-1} \mathbf{C}^{T} \mathbf{k}
$$

Of course the exact condition would be associated with a nonlinear relation involving additionally higher-order terms in the elements of the vector $x$. This relation can now be sub: tituted into Eq. (19) to eliminate the explicit dependence of the trial energy on p.

$$
E^{\mid 2\}}(\mathbf{x})=E(0)+\mathbf{w}^{T} \mathbf{x}+\frac{1}{2} \mathbf{x}^{T}\left(\mathbf{B} \cdot \mathbf{C} \mathbf{M}^{-1} \mathbf{C}^{T}\right) \mathbf{x}
$$

The matrix (B-CM- ${ }^{-1} \mathbf{C}^{T}$ ) is called the partitioned orbital hessian matrix. Conceptually, it is the second denvative of the eigenvaiue of the hamiltonian matnx with respect to pairs of orbital rotarions. The matrix elements of B. by contrast, are the second derivatives of the expectation value of the hamiltonian matrix with respect to pairs of orbital rotations for the fixed reference CSF expansion vecior $\mathrm{Cl}^{\circ}$. The approxumate energy expression Eq. (2 subspace, or as an approximation to Eq. (20) valid for small orbital variations.

Using Eq. (22) in an iterative manner, it is possible to minimize the trial energy with respect to both orbital variations and CSF coetficient variations. The MCSCF wave function is then determined by the features of this energy surface. This will be called the "minimum-energy" definition of the MCSCF wave function. A somewhat surprising resul is that minimization of an arbitrary MCSCF hamiltonian eigenvalue with respect to orbital

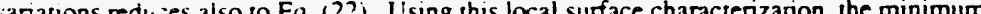
variuns Suppose hower, that for a given ser of orbin it is possible 10 compure states 1.7 .

e some wave function that is judged to be more accurate than the truncatec MCSCF expansion. In practuce. this might be, for example, a large-scale muldireference $\mathrm{Cl}$ wave function based on the MCSCF expansion space: in principle, it could be the exact full-CI wave function. In this wave function, the individual CSF mixing coefficients will assume slighdy different values from those of the lowest eigenvector of the MCSCF hamiltonian matrix. This could be regarded as a relaxation effect of the reference wave function vector. When expanded in overlap between these iwo vectors may be characterized using the quantity $\operatorname{Cos}(\theta)=\left(\frac{c^{\text {full }} \mathrm{Cl}}{c \mid \mathrm{c}^{\mathrm{full}} \mathrm{Cl}_{1}}\right)$ This overlap with the exact wave function may also be used to characterize the MCSCF wave function. The "maximum overlap" vector within the MCSCF expansion space, defined as MaxiCos( $(\theta))$, is given by

$c_{j}=c_{m}^{\text {tull-Ct }}$ where the mapping vector $m$ associates the MCSCF expansion CSFs with the full-Cl expansion CSFs. For ground state calculations, the expectation value of this vector will be higher than the MCSCF-eigenvalue choice; for excited states, it may be either higher or lower since it is not necessarily orthogonal to any lower-energy MCSCF eigenvectors. In principal, it would be possible 10 maximize this overlap wich respect to orbital variations principa in would be posible choice of orbitals, the expansion coefficients of the exact wave function do in fact depend on the orbitals. Although this maximum-overlap concept has limited practical value. it defines an alternative to the energy-minimization definition of the MCSCF wave function. Other conditions could also be chosen to define the MCSCF vector $c$, including, for example, perturbative expansions or approximate solutions to various time-de-endent formulations.

In summary, an essential feature of the MCSCF wave function is the simultaneous variations of the orbials and expansion coefficients. In essentially all other electronic

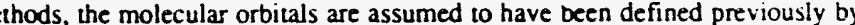
some method. In practice, this is a (single configuration) SCF method, or, as is occurring more and more frequendly, a more general MCSCF method. In this way, an understanding of the MCSCF method is essential to, and a basis for, the understanding of many other electronic structure methods.

\section{THE MULTIPLE SOLUTION PROBLEM}

In most cases the MCSCF optimization procedure based on energy minimization results in a well-defined energy and wave function at each molecular geomeory. We now examine the behavior of the MCSCF energy and wave function where two distinct wave function solutions may be found. The cases examined here are characterized by a relatively abrupt change of the orbitals or of the CSF expansion coefficients with a small change in some exiernal variable. This external variable is usually a continuous molecular geomerry some exiernal variable. This external variable is usually a contunuous molecular geometry
change, but generally this discussion applies also to changes in the strength of some applied change, but generally this discussion applies also to changes in the strength of some applied
field, or even a continuous change of some orbital basis function parameter (e $g$ an orbital exponent or exponent scale factor). We herein assume that the extemal periurbation is a geometry change. since this is probably the most common occurrence of multuple solution problems. The multiple solution problem is usually discussed in the context of "rootflipping" problems for excited state optimization. The multiple solution problems associated with single-state, and in particular the ground state, cases will be emphasized primarily in this section. Consider the behavior of the MCSCF energy as a function of two coordinates, the external perturbation and one internal degree of freedom. characterized as

$|\lambda: R\rangle=\exp (K(\lambda: R)) \exp (P(\lambda: R))|0: R\rangle$

By this notation it is implied that for each geometry $R$, there exists a: least one value for $\lambda$ which satisfies the MCSCF optimization conditions. At some values of $R$, there may exist more than one MCSCF solution, and each solution is associated with a distinct value of the parameter $\lambda$. These multiple solutions could correspond, for exampie, to distinct covalent and ionic descriptions of a polar molecule, or to two distinct, symmetry-related descriptions of an electronic state or to two distinct correlared descriptions of a single electronic state. The contour plot of Fig. I shows a schematic representation of the behavior of the energy is a function of these two degrees of freedom.

Consider first the path marked $A$ in Fig. 1. At small $R$, the energy minumum is unique and is located in the upper left of the graph, corresponding to irge- $\lambda$ values. The minimum energy path intersects the points at which the contour lines are exactly vertical 
th veen in the graph. there is no reason why individual contour lines must have such : eracil slope, and as the path $A$ progresses touards larger $R$ values, It is seen to end abruptly at point $A_{1}$ ) The contour lines associated uith larger $\mathbf{R}$ values in the neighborhood of point $A_{0}$ do not have a vertical slope. Consequents, the smoorh path defined by this sequence of points of verical energy contour slope varishes in this neighborhood beyond this critcal point. Sumilarly, path B is a distunct munimum-energy path that begins in the lover nght of the graph and progresses to higher energy regions of this suriace unul it ends abruptly at point $B_{0}$. It is clear fr Recall find $B$ do not intersect.

Recall from the discussion in the previous section that the ground state MCSCF energy may he defined as that which minimizes the lowest hamiltonian eigenvalue with respect to orbital vanatuons. For a given orbital set, the lowest energy solution, corresponding to $c_{p p}$ of $\mathrm{E}_{4}$ 120). may be determuned distinctly and unambiguously as the lowest eigenvalue of

\section{$E(\lambda, \mathrm{R})$}

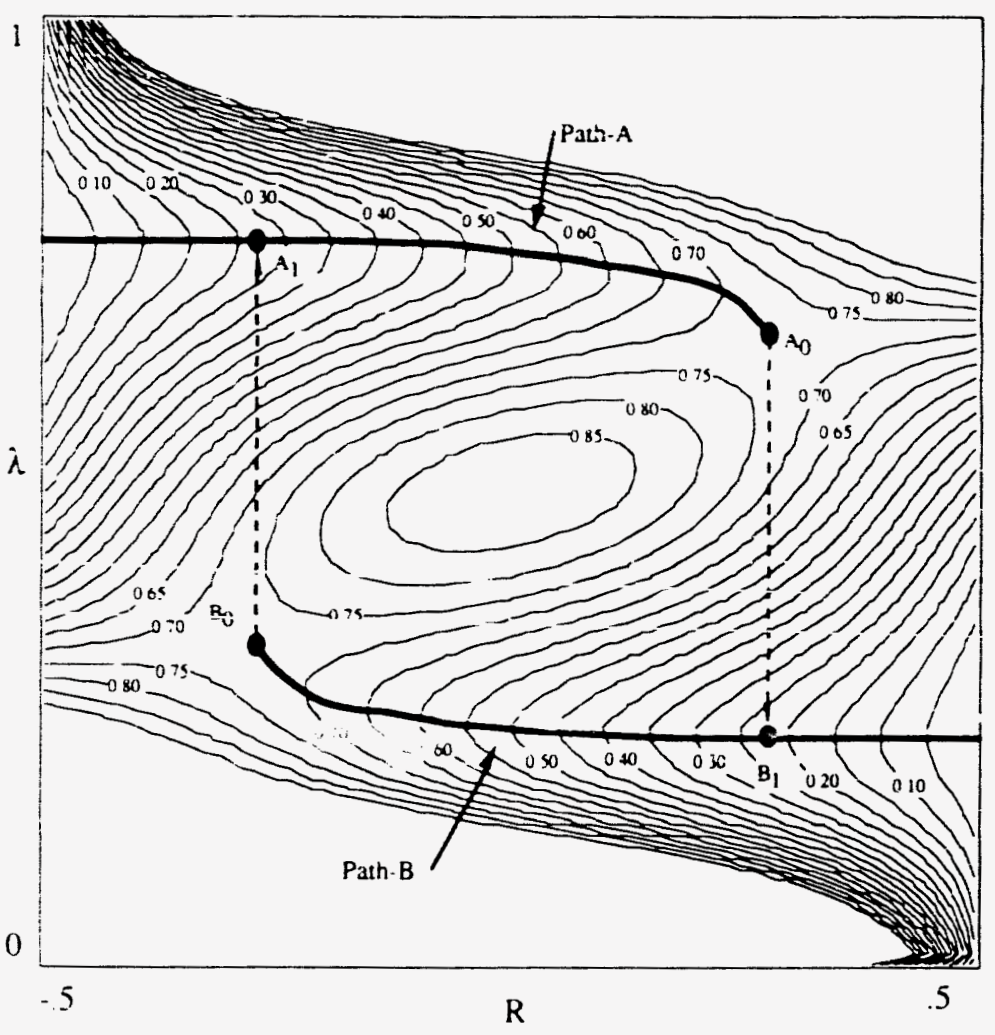

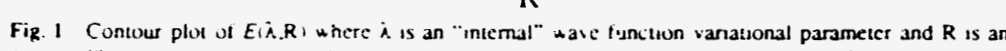

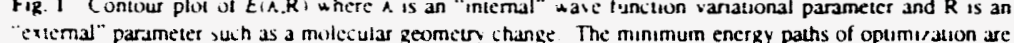
dinived as past $\mathrm{A}$ and path $\mathrm{B}$ the hamiltonian matrix. However. different orbital choices will ledd to different hamilionian matrices, and the minimum of the energy with respect to orbital vanations is only a local charactenzation of $E(K)$. As a function of $R$, this local characterization of the MCSCF wave function is not guaranteed to be continuous everywhere. With a careful parameterization of $K(\bar{\lambda}, R)$ and $P(\lambda, R)$, a situation similar to that depicted in Fig. I can be recognized.

Recall however, that the usual description of the Born-Oppenheimer PES does not involve $\lambda$-type coordinates (but rather geometrical coordinates only), and that at a fixed geomerry the wave function optimization problem does not involve geomerical coordinates (but $K$ and $P$ coordinates only). It is instrucrive to consider the consequences of the general energy surface shown in Fig. 1 from these two, more familiar, points of view Consider first the PES behavior for large values of $R$. The computed MCSCF energy correspunds to the energy along the path B in Fig. 1. If small steps are taken along the $R$ coordinate, and if the initial MCSCF wave functions in the optimization process are chosen to be those of the nearby point, then smooth convergence behavior will be observed all the way to the cighe to the lo collapse to the lowe discontmuity of the energy curve $E(R)$ as shown in Fig. 2 . Hopefully, the abrupt energy change will be large enough to draw attention, but there is no guarantee that this must occur since this depends on the quantitative details of the energy surface rather than the qualitative aspects discussed here. Having observed an abrupt energy change. furher examination reveals the nature of the corresponding abrupt change of the wave function (i.e. in the orbitals and/or CSF coefficients). The stepping procedure could now be reversed, from small values of $R$ to larger ones. This time another smooth curve is computed along path $A$ from point $A_{1}$ to the neighborhood of point $A_{0}$ of Fig. 1. As before, the wave function optimization at each new geomery is assumed to star from the converged wave function of the nearest point on the path. Smooth and rapid convergence will usually be observed, past the intersection point IMCSCF in Fig. 2, until, as before, the energy collapses suddenly at the critical point $A_{0}$ back to the $B_{1}$ point on the onginal path

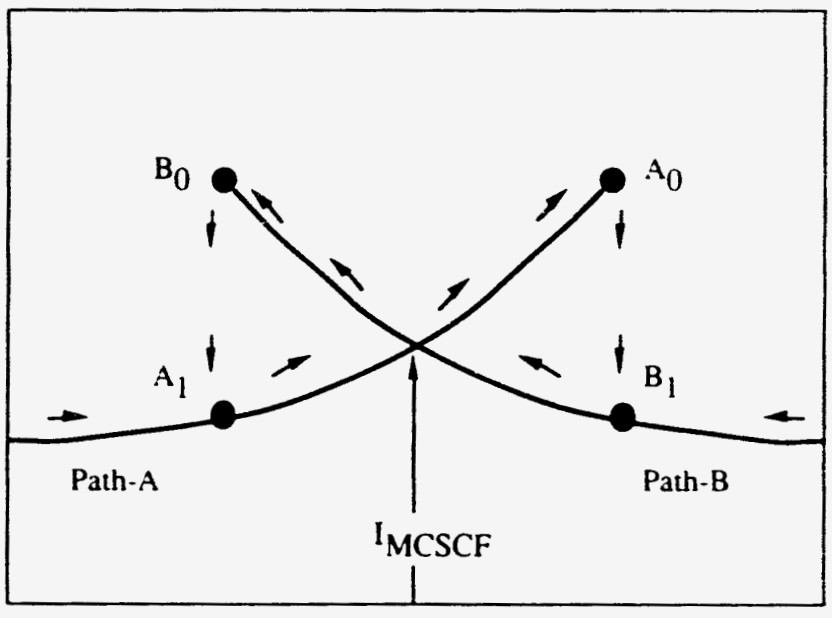

$\mathrm{R}$

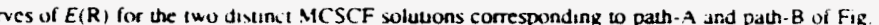
The "figure- 8 " loop corresponds to the provection of the hysteresis path of Fig 1 
in Fig 1 This pru, edure can be repeated indetinitely, and with arbitranly small geomery seeps. and as long ts the wave function optimization procedure is directed toward local energy minimization, and as long as a sufficiently accurate mal wave function is chosen for the optimization step. this hysteresis thavior will be observed When projected onto the $E\{R$, graph of Fig 2. this appears to te 3 rather puzzling "figure 8 " pantem.

Conster next the wave function optr..zatuon for specific values of $R$. For this value of $R$ there is a single energy munimum as large $\lambda$. As $R$ increases, a new high-energy minimum appears at small $\lambda$ in addition to the global minimum at large $\lambda$. At intermediate values of $R$, both minima are seen to be comparable in energy value. At larger values of $R$. the small- $\lambda$ minimum, ine global minimum, and the large- $\lambda$ minimum eventually becomes stmply an inflection point (specifically a stationary point with zero second denvative) and then vanishes. These inflection points correspond to the critucal points $A_{0}$ and $B_{0}$ of Fig. 1 .

Vore in passing that the overall symmeny displayed in Figs. 1-3 is consistent with two symmery broken descrptions of an electronic state, with $R=0$ being the point at which the atomic nucle: assume a symmetric position. The two equivalent munima in the $E(\lambda)$ curve

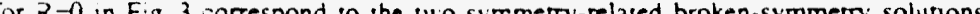
Orher cases. such as compeing covalent and ionic descripions of a molecular state mentined prevously, would nor likely display the overall symmeny or Fig. 1. An altempt sescription of any such multiple-solution situation. See Ref. 9 and references therein for

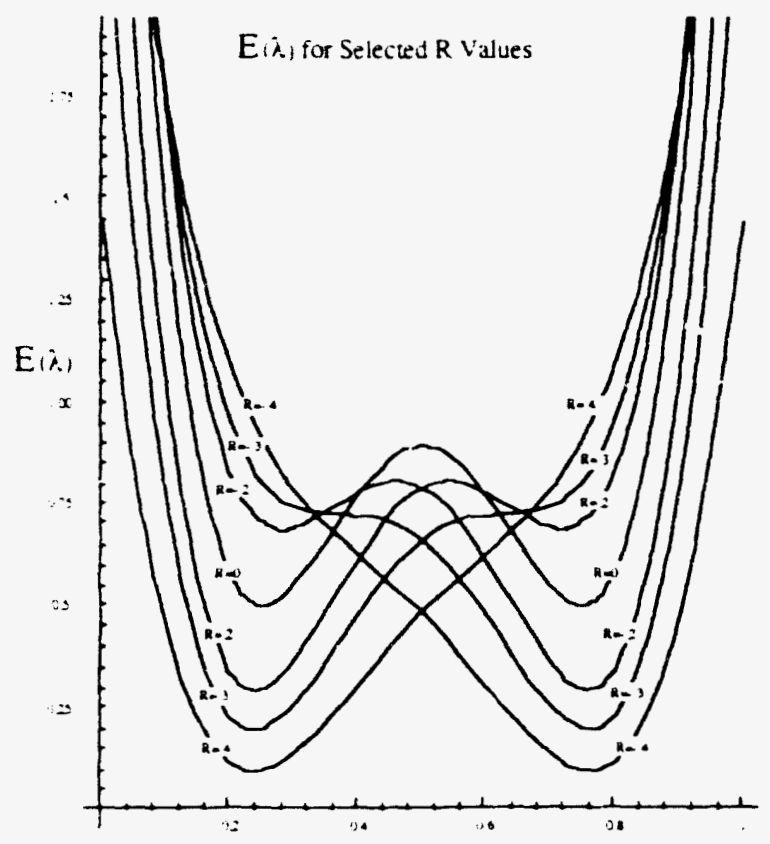

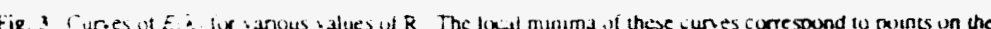

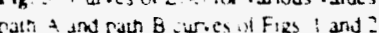

specific discussions of the symmetry-breaking problem for small hydrocarbon radicals

Of course there are many other similar situations that could be observed. The question then arises as to when, and if the definition of the wave function should change from one MCSCF minimum solution to the other. Consider first the situation depicied schematically in Fig. 4. This situation is analogous to that given previously in Fig. 2. but now three new energy cunes are added. an exact (full-CI) curve, and two approximate curves, one with ach of the possible sets of MCSCF orbitals. The full-Cl curve is independent of the orbital choice, so this curve could in principle be computed with either choice of orbital sets. This curve is depicted schematically as a smooth curve, characieristic of an avoided crossing. but essentially any kind of behavior could arise, even the cusp behavior associated with allowed state crossings of polyatomic molecules. This exact energy curve is usually not available for examunation, but must be inferred from the vanous approxumate curves.

In order for the MCSCF curve to be continuous in R, it is clear from Fig. 4 that we must switch wave functions exactly at the point IMCSCF. Any other switching point would mest in a discontinuus MCSCF energy Curve. Even an inis swilching point. ciscis we function once the energy coninu. crondidon heen imposed.

it is typical however to use the MCSCF method simply for defining the orbitals of a more accurate wave function. In Fig. 4, an $\mathrm{MRCl}$ wave function is used as an example of such a wave function 10 . In general, any wave function which is deemed to be more accurate than the MCSCF wave fu iction could be used for this illustration, including those determined using contracted $\mathrm{Cl}$ methods $11-13$, multireference $A C P F$ methods ${ }^{14}$, multireference coupled-cluster methods ${ }^{15-17}$, or multireference perturbation theory methods 18-22. All such methods have the characteristic that they depend on the orbital

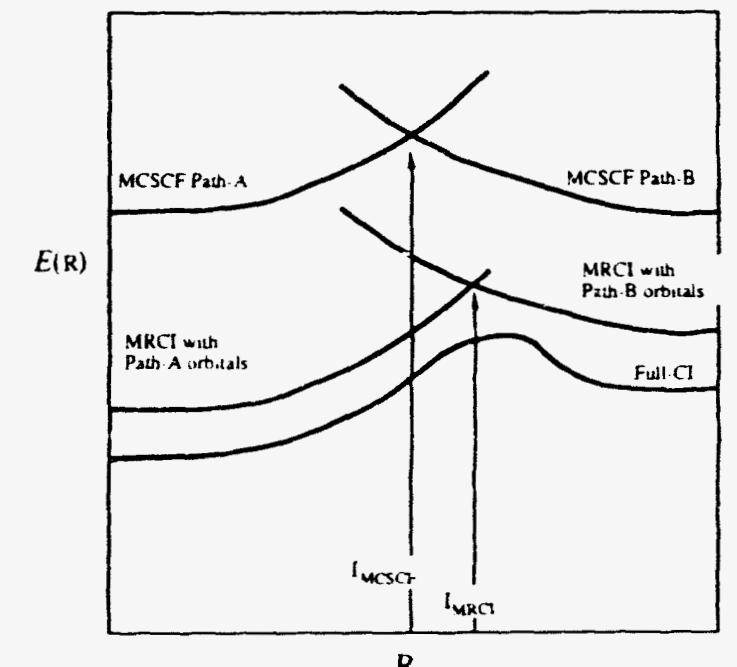

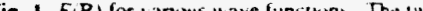
uxed in the iwo MRCl curves the full Cl iuree is independent of the orbical thoke 


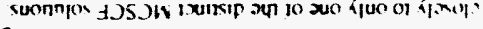

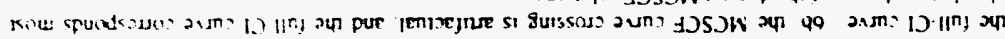

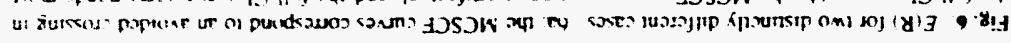
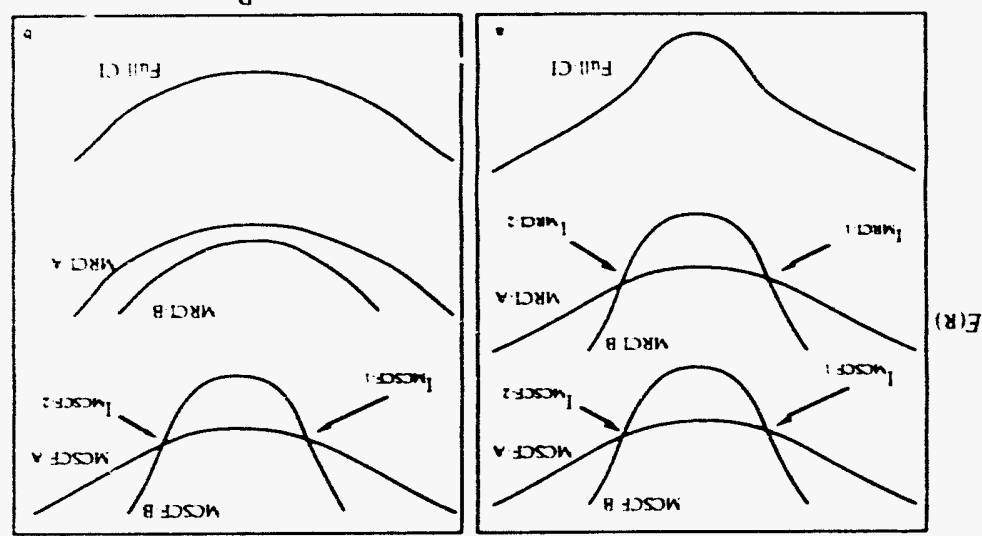

sanjes posipasd

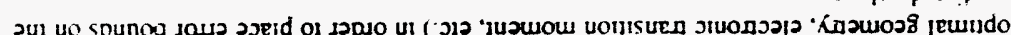
.

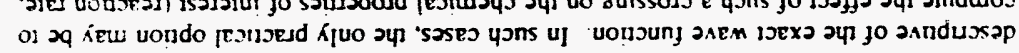

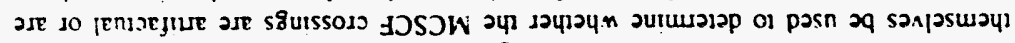
fa jouve y

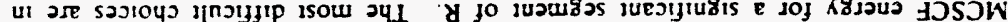
is 2 nol 24 s.ss

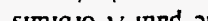
sद्व is $2 \mathrm{q}$ गद deprs.

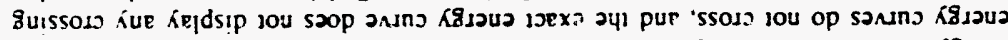

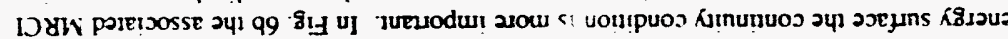

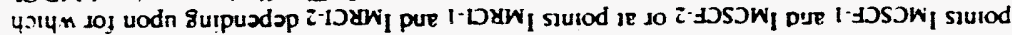

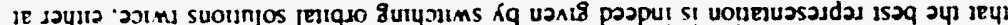
í

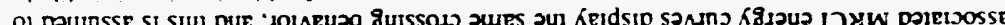

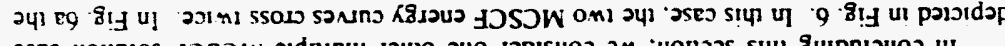

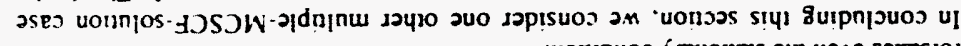

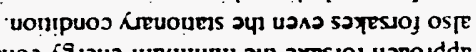

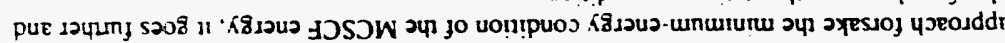

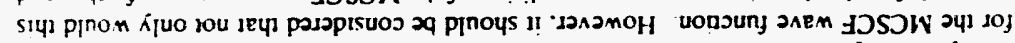

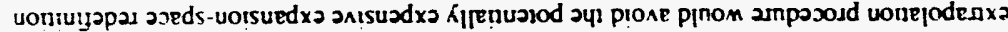

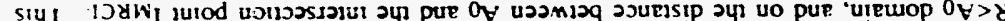
د4 sict jo or

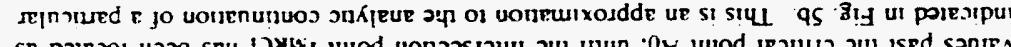

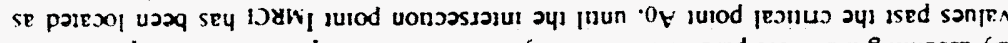

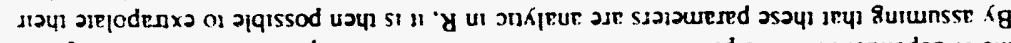

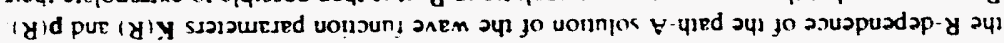

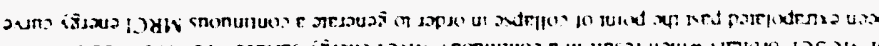

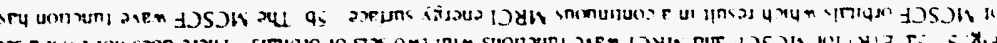

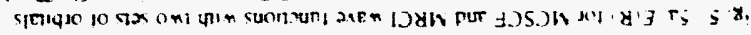
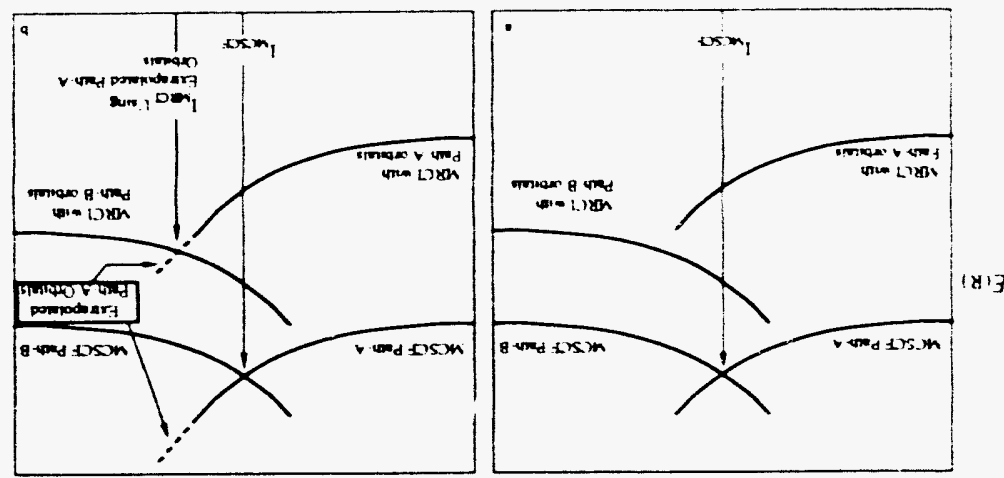

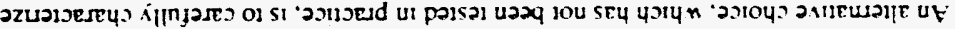

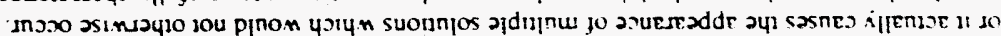

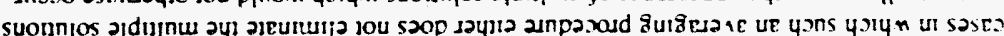

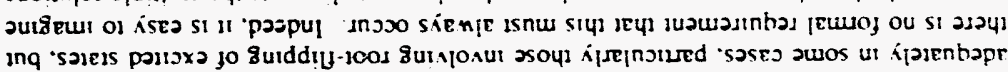

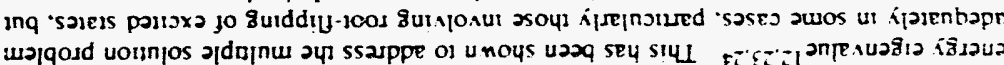

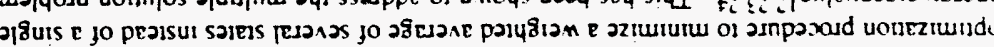

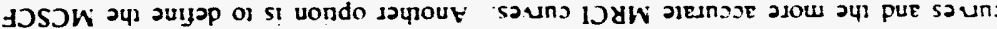

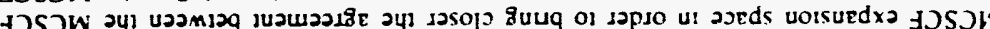
IJSכW 保

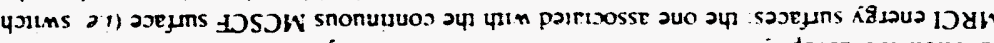

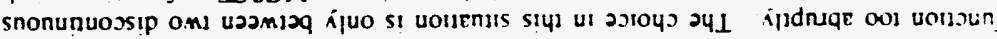

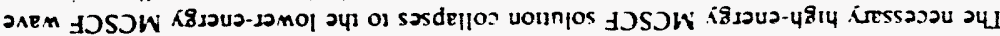

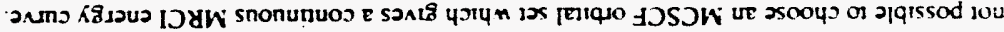

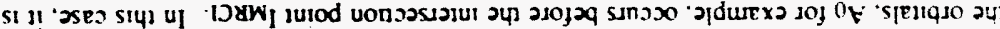

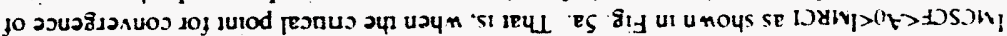

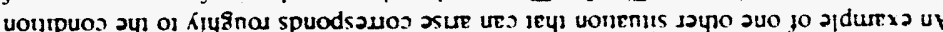

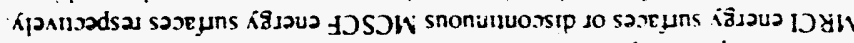

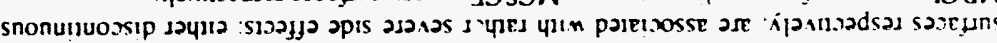
K8sدu 1 J 250Y] 's

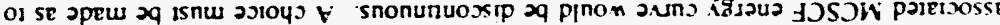

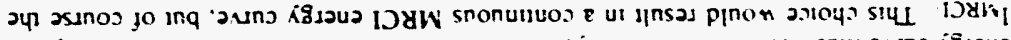

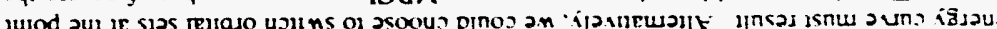

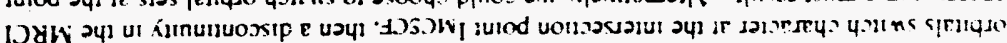

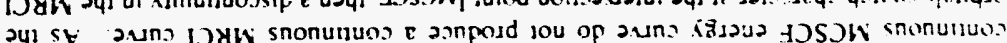

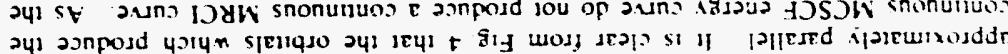

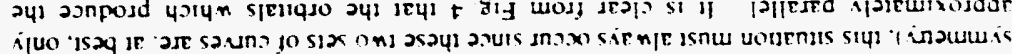

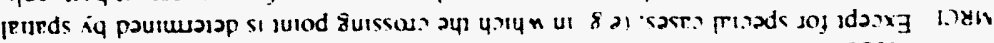

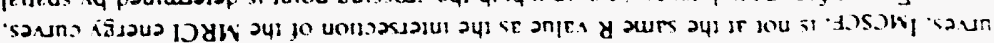




\section{THE METHOD OF TRIGONOMETRIC INTERPOLATION}

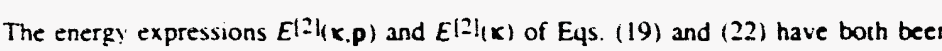
used in the MCSCF optimization process'. the former being associated with those optimization methods based on the wave function hessian matrx and the latter being associated with those involving the parationed orbital hessian matrix. In this section, a recently introduced wave function opumization method. called multidimensional ingonometic interpolavion's, is described brefly. and the relavon or this method (19) and (22) is discussed.

The basis of this interpolation method is understood most easily by considering the dependence of the MCSCF urial energy as a function of a single orbital rotation variable. In this special case. the energy depends on (the scalar) $x$ through the orbital transformation matrix $\mathrm{L}$. The appropriate $2 \times 2$ subblock of this marrix may be writien as

$$
U(\alpha)=\exp (K)=\left(\begin{array}{cc}
\cos (\alpha) & -\sin (\alpha) \\
\sin (\alpha) & \cos (\alpha)
\end{array}\right)
$$

which is clearly periodic in $x$ with period $2 \pi$. For further analysis, either of the two types of energy expressions discussed previously might be used: trial energy expressions of the type Eqs. (10-14), which are appropriate for considering independent variations of the orbutals and CSF coefficients, or expressions such as $\mathrm{Eq}$. (20), which are appropriate for considering orbital rotanions as independent varables and the CSF coefficients as implicit dependent $、$ anables. The work to date using the trigonometric interpolation approach has concentrated on the latter choice due to the following simplification. Observe first that $U(x+\pi)$ is related to $U(x)$ according to the ungonometric addition relations

$$
\begin{aligned}
U(x+\pi) & =\left(\begin{array}{c}
\cos (x+\pi)-\sin (x+\pi) \\
\sin (x+\pi) \cos (x+\pi)
\end{array}\right) \\
& =\left(\begin{array}{c}
\cos (x)-\sin (x) \\
\sin (x) \cos (x)
\end{array}\right)\left(\begin{array}{cc}
\cos (\pi) & -\sin (\pi) \\
\sin (\pi) \cos (\pi)
\end{array}\right)=\left(\begin{array}{cc}
\cos (x)-\sin (x) \\
\sin (x) \cos (x)
\end{array}\right)\left(\begin{array}{cc}
-1 & 0 \\
0 & -1
\end{array}\right) \\
& =-U(x)
\end{aligned}
$$

In other words. the two orbitals simply change signs at $(x+\pi)$ relative to their values at $x$. The tnal energy of Eq. (20) does not depend on orbital phases since the elements of $c_{\text {apr }}(\kappa+\pi)$ must compensate exactly for any sign changes relative to $c_{\text {opr }}(\kappa)$. The trias energy of Eq. (20) must therefore be periodic in $x$ with period $\pi$. Consequently, $E^{\text {ir:al }}(\kappa+\pi)=E^{\text {trial }}(\kappa)$ under this assumption of optimal CSF expansion coefficients. Clearly truncated energy expressions such as $E^{[2]}(\boldsymbol{x}, p)$ and $E^{[2]}(\boldsymbol{k})$ do not reproduce this periodic behavior. and therefore are valid only for small values of the paramerers $x$.

The next problem is how to use this insight into the periodic nature of the trial energy expression of Eq. (20) in order to improve the behavior of an approximate energy expression. There are many ways that this could be done; the following approach build upon some of the previous experience in using truncated energy expressions such as $E^{[2]}(x)$ of Eq. (22). Again taking a one-dimensional case as a model, the truncated energy expression has the form

$$
E^{|2|} \mid(x)=E(0)+w k+\frac{1}{2} B x^{2}
$$

where it is assumed that the energy. gradient, and hessian are known at the reference point, $\kappa=0$. We now $u$ ish to use this information not only at $k=0$, but also at $x=\pi, k=2 \pi, \ldots$ The general idea is that by imposing this periodic condition on the tral energy function. It will more closely mimic the toue tra "nergy funcion and will be useful for a larger domain of $\therefore$ values. In a sense. we are interpolating the unknown trial energy function between the
Infinite number of points $x=10, \pi, 2 \pi \ldots)$ at which certan information is known. The strategy used is to replace the independent variable $x$ with a periodic function $f(x)$ This automatically results in a periodic interpolating function having the form

$$
E^{r I F}(\mathrm{~K})=E(0)+w \mathrm{f}+\frac{1}{2} B \mathrm{f}^{2}
$$

Differentiating this expression with respect to $\mathrm{x}$ gives

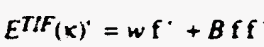

$$
\begin{aligned}
& E^{I I F(x) "}=w \mathrm{f}^{\prime \prime}+B \mathrm{ff}^{\prime \prime}+B\left(\mathrm{f}^{\prime}\right)^{2}
\end{aligned}
$$

where the derivatives are denoted with primes. In order for $E^{I I F}(0)=E(0)$ to be sansfied for arbitrary $w$ and $B$, it follows that $f(0)=0$ must be sarisfied Similarly, for $E^{T I} F_{(0)}=w$, is follows that $f(0)^{\prime}=1$. ions of the form $f(x)=\frac{1}{2} \operatorname{sen}(2 n x)$ (or linear combinations thereon) satisfy all of these conditions and also display the correct periodicity. The simples of these is the function consisting of the single term $f(x)=\frac{1}{2} \sin (2 x)$. In the one-dimensiona. case, this results in the interpolating function

$$
\begin{aligned}
E^{T I F}(x) & =E(0)+\frac{1}{2} w \sin (2 x)+\frac{1}{8} B \sin ^{2}(2 x) \\
= & E(0)+\frac{1}{2} w \sin (2 x)+\frac{1}{16} B(1-\cos (4 x))
\end{aligned}
$$

The second form shows clearly that the gradient contributes to a component of the interpolating function that possesses sinusoidal behavior of period $\pi$, whereas the hessian term contributes a component with period $\pi / 2$.

In the general multidimensional case, the functional form for the coordinate transformation is taken tc be

$$
F(K)=\frac{1}{4}(\exp (2 K)-\exp (-2 K))=\frac{1}{2} \sinh (2 K)
$$

The matrix $\sinh ($ ) function appears instead of the $\sin ()$ function because the skeu symmetric marix $K$ has purely imaginary eigennalues. The vector $f$ is now defined to be the unique elements of the skew-symmetric marnx $F(K)$ (in the same way that $\mathbf{X}$ is reiated to $\mathbf{K}$ ). The multidimensional interpolating function is then writen explicidy as

$$
E^{T I F}(\mathbf{x})=E(0)+w^{T} \mathbf{f}+\frac{1}{2} f^{T}\left(\mathbf{B}-\mathbf{C M}^{-1} \mathbf{C}^{T}\right) \mathbf{f}
$$

Conceptually, MCSCF wave function optimization using this approach consists first of determining the energy, gradient, and hessian (i.e. $E(0), w, B . C$, and $\mathbf{M}$ ) using the curren trial wave function. Then using these matrices, the corresponding $E^{T I} f_{(x)}$ is optimuzed with respect to the orbital rotation parameters $\mathbf{K}$. New orbitals are defined using the transformation $\exp (K)$, and new CSF expansion coefficients are determined from the MCSCF hamitonian eigenvalue equation. This process is repeated until convergence is obrained 25 For practical reasons, it is useful to consider optimization procedures that do obtained ${ }^{25}$. For practical reasons. it is useful to consider optimization procedures that do not require the explicit computation and storage of the hessian matr $x$ blocks $\mathbf{B}, \mathbf{C}$, and $\mathbf{M}$
With this goal in mind, it is possible to design procedures that optimize $E^{\text {TiF }}(\mathbf{x})$ while requiring only the computation of matnx-vector products of the form Bx. $\mathbf{C}^{T} \mathbf{x}, \mathbf{C y}$. and $\mathbf{M} \mathbf{y}$ for subspace expansion vectors $\mathbf{x}$ and $\mathbf{y}$ Such matrix-vector products may be computed in several ways, depending on the relatuve costs of hessian matr.x construction. partia! integral iransformations, hamiltonian matrix-vector products, and transition density matrix construction 1.6.12.26. Since these operations are required already in most second otder 
MCSCF optumization schemes ${ }^{1}$. the tngonomeonc interpolatuon approach may be added to most existing programs as an option with only relatively minor modifications.

The global behavior of the $E^{I I F}(x)$ interpolatung function may be seen in Fig. 7 These energy curves are one-dimensional stices from the muludimensional energy surface. This line is determined by a constant direction marrix $\Delta$ and the scalar parameter $s$ as $K=\Delta \Delta$ (see Reference 25 for further details). Two other approximate energy curves are also displayed Eq. $(22)$ and the corresponding $a U$. has been used successfully for accelerating the convergence of MCSCF wave function optimizanion. Observe that $E^{\text {thal }}(\boldsymbol{x})$ is periodic with period $\pi$ as discussed above. When the expansion poin: $t_{0}$ is close to the energy minimum, as in Fig. 7a, then all three approximating curves predict accurately the location of the actual minimum. In fact, all three curves are correct through second order and lead io the same asymptotic convergence behavior.

As seen in Fig. $7 b$, as the reference point is moved further away from the minimum. the truncated expansion $E^{[2]}(x)$ fails to predict the location of the energy minimum. The Feriodic $E^{\Pi I F}(x)$ function and the rational function approximanon $E^{P S C I}(x)$ both still approximate the minimum positon reasonably well. As the reference point is moved even furher away from the minimum as in Fig. 7c, the penodic $E^{I I F}(\mathbf{x})$ is the only approximate function that predicts the position of the minimum. Since this region of the curve has negative curvature, the truneated $E^{\{2 \mid}(\mathbf{x})$ curve has no minimum. The high-energy local minimum of the rial energy curve is also predicted by the $E^{T I F}(\mathbf{x})$. Finally in Fig. 7d the reference point is moved to a neighborhood of a maximum. Again, only the $E^{T I F}(x)$
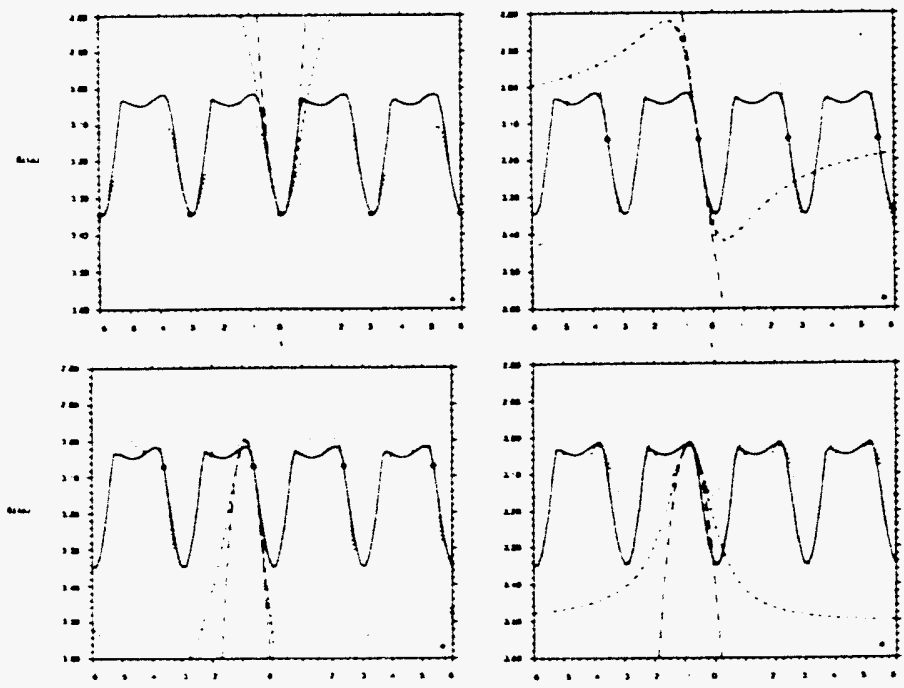

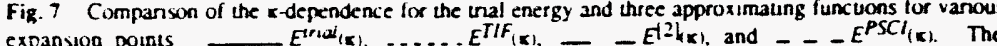
expansion poins $10:$ are 110 . 0.5. 0625 . and 084 for figures a-d respectively along a line in the muludimensional orbical varusuonal space function approximates the positon of the energy minimum. Due to the small gradient at the expansion point, the interpolating curve is dominated by the period $\pi / 2$ contributions from the hessian as discussed above. These figures demonstrate that the $E^{I I F}(\mathbf{K}$ interpolating function is capable of approximating the features of the true trial energy function even when the reference wave function is not in the region of the opumal solution

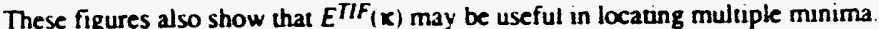

In addicion to displaying the correct periodic behavior for individual orbital rotauons. (is 25 . These of sets of mutrially commuting orbital rotation operators, and along certain lines in the multidimensional variational space called "direction patterns" which are related to the eigenvectors of the matnx K corresponding to specific eigenvalues. Qualitatively correct behavior is also displayed for redundant orbital rotations; namely, both $E^{I I F}(\mathbf{x})$ and $E^{\text {mal }}(\mathbf{x})$ are independent of such orbital ransformations.

\section{Acknowledgments}

The author wishes to thank Professor G. L. Malli, director of the NATO ASI "Relaivistic and Electron Correlation Effects in Molecules and Solids". Vancouver, B.C. Canada. August 1992, for the invitation to present this matenal. This work was supported by the U.S. Deparment of Energy. Office of Basic Energy Sciences, Division of Chemical Sciences, under contract W-31-109-ENG-38.

\section{References:}

I. R. Shepard, in: "Ab Insto Methods in Quanwum Chemiscry II. Advances in Chemical Physics 69", K. P Lawley, od.. (Wiley. New York, 1987) pp. 63-200.

E. Dalgaard and P. Jergensen. J Chem Phys 69. 3833 (1978)

E Daloard Chem Phys Lets. 05.559(1979).

D L Yeager and P. Jorgensen J Chem Phys 71.755 (1979)

5. D. Thouless. The Quanum Mechanics of Many-Body Sysems." (Academic Press. New York. 1961). (see in parucular pp. 25-27)

6. J. Olsen, D. L. Yeager, and P. Jorgensen. Adv. Chem. Phys. $\$ 4,1$ (198)

7. R. Shepard, L. Shavitu, and J. Simons. J. Chem. Phys.. 76. 543 (1982).

8. H J. Aa Jensen. P. Jergensen, and H Agren, J Chem. Phys. 87.451 (1987).

P. G. Szatay. A. G. Cószarr. G. Fogarass, A. Karplea, and H. Lischika. J Chem Phys. 93. 1246 (1990).

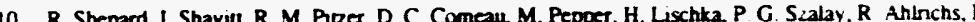
B. Brown, and J.G. Thas. Int I Quartum. Chem. S22. 149 (1988).

P E. M. Siegbahn. Chem. Phys. Lett. 119. 515 (1985).

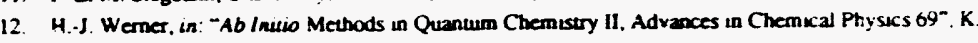
P. Lawky. od.. (Wiley. New York, 1987) pp. 1-62.

13 H.J. Werner and P. J. Knowles. Thear Chum Acta 78. 175 (1990).

14. R. Ahrichs and P. Scharf, in: "Ab Iniao Methods in Quantum Chemissyy l. Advances in Chemical Physics 67". K. P. Lawley. ed. (Wiky. New York. 1987) pp. 501-537.

15. A. Banerjoe and I. Simons. Ins I Quantum Chem. 19. 207 (1981).

16. W. D. Laxilg and R. J. Bartett, Chem Phis Lets. 104. 424 (1984)

17 M R Hoffmann and I Sumuns. I Chem Phrs 90.3671 (1989)

18 M. A. Anderson and R. J Cave. Chem Phvs 15s. I (1991)

19 B O. Roos. K. Anderson. and M. P. Fulscher. Chem. Phys. Lets. 192.5 (1992)

2) E R. Davidson and C. F. Bender. Chem Phvs Leal 59. 369 (1978)

II R B Murphy and R P 'Aessmer I Chem Phus. 97.7170 (1992). 


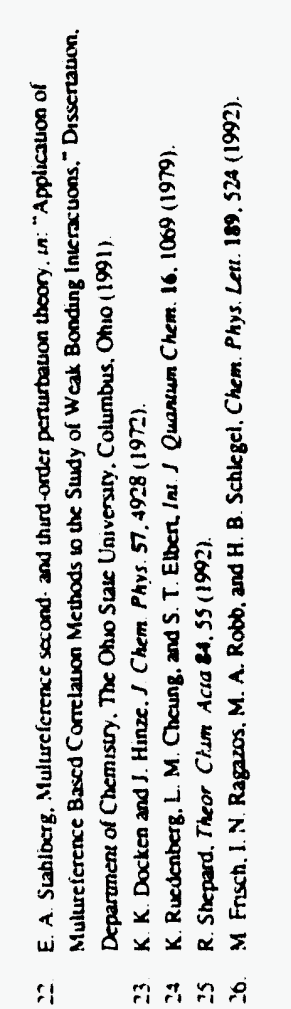



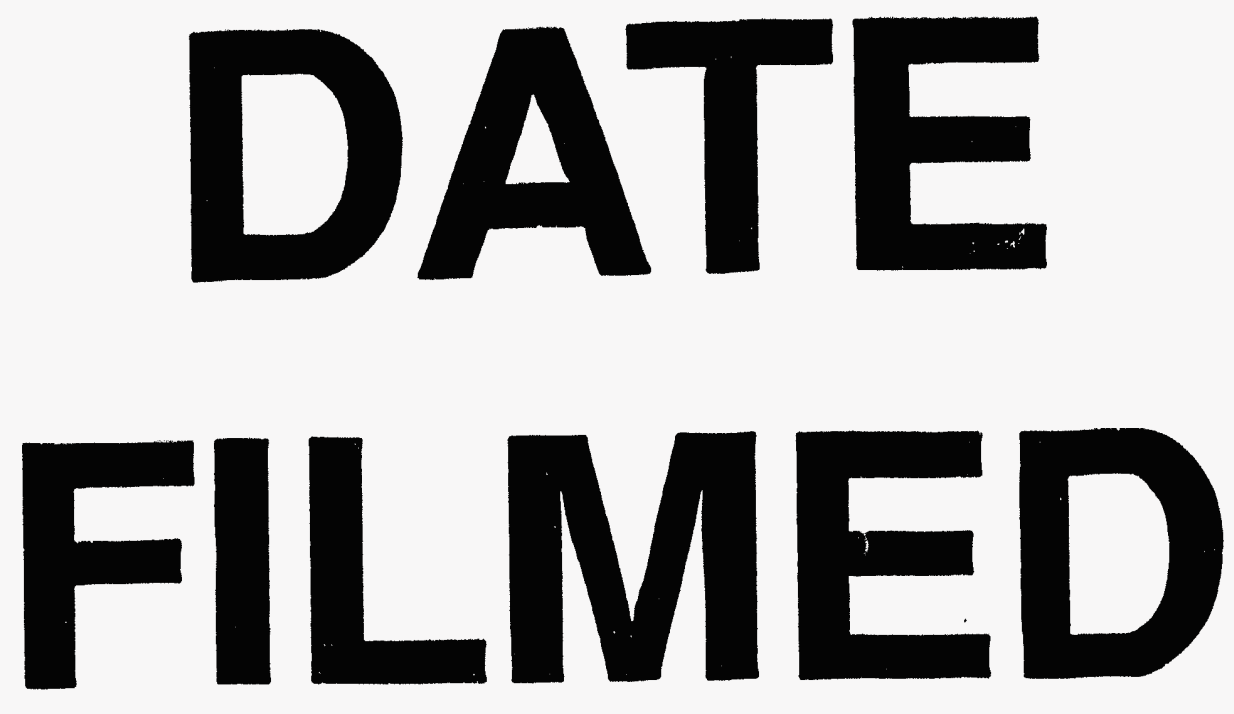

$11 / 17 / 93$
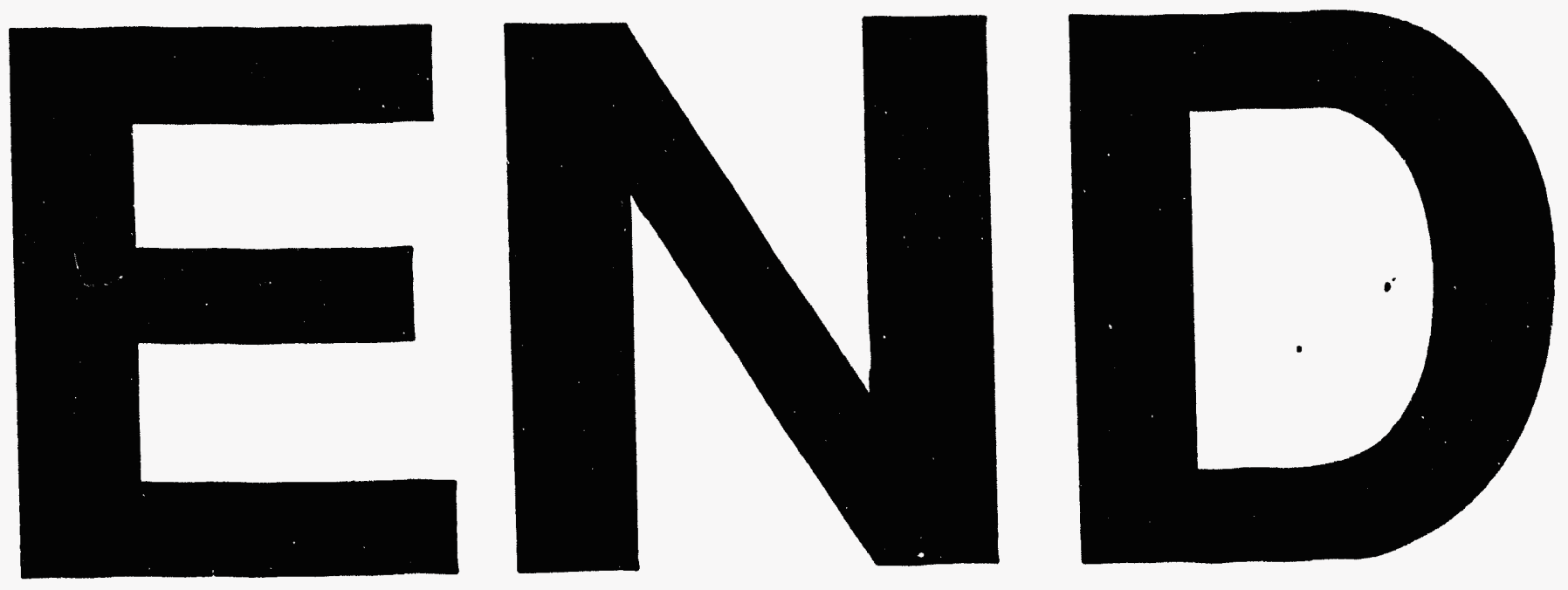


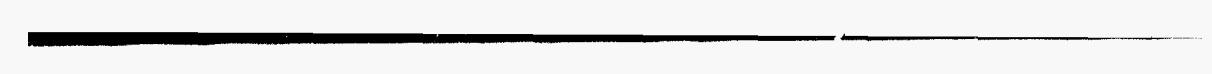

